\title{
Analysis of Risk Factors of Fever among Inpatients in Rehabilitation Department Based on Lasso Regression Analysis
}

\author{
Ling Liu1*, Jiayi Wang1*, Yingxue Zhong1, Chunhua Wang1, Yu Chen1\#, Yitao Mao#, Zhiwei Mou1\# \\ ${ }^{1}$ Department of Rehabilitation Medicine, the First Affiliated Hospital of Jinan University, Guangzhou, China \\ ${ }^{2}$ Department of Radiology, Xiangya Hospital, Central South University, Changsha, China \\ Email: "mouzhiwei@jnu.edu.cn
}

How to cite this paper: Liu, L., Wang, J.Y., Zhong, Y.X., Wang, C.H., Chen, Y., Mao, Y.T. and Mou, Z.W. (2020) Analysis of Risk Factors of Fever among Inpatients in Rehabilitation Department Based on Lasso Regression Analysis. Journal of Biosciences and Medicines, 8, 122-131.

https://doi.org/10.4236/jbm.2020.85012

Received: April 9, 2020

Accepted: May 6, 2020

Published: May 9, 2020

Copyright $\odot 2020$ by author(s) and Scientific Research Publishing Inc. This work is licensed under the Creative Commons Attribution International License (CC BY 4.0).

http://creativecommons.org/licenses/by/4.0/

\section{(c) (i) Open Access}

\begin{abstract}
Purpose: To explore the fever-related risk factors of inpatients in Rehabilitation Department, and to provide reference for patients with high risk of fever to take corresponding nursing measures. Methods: The study was conducted on the Rehabilitation Department of The First Affiliated Hospital of Jinan University from July 2019 to December 2019. The fever group included 51 patients and the non-fever group included 49 patients without fever. The two groups of clinical data, comorbidities, related laboratory values, possible risk factors of fever were analyzed by case regression analysis, and the relevant risk factors were screened out by LASSO (least absolute shrinkage and selection operator) regression analysis. Results: According to the results of Lasso regression analysis, pressure sore or skin infection, history of hypertension, current history of respiratory tract infection, feeding patterns were the higher risk factors of fever in inpatients in Rehabilitation Department, while the first course of disease, main diagnosis, history of respiratory tract infection within half a year, kidney damage and hospitalization days were lower risk factors. Conclusion: This study is helpful to early identify the fever risk of inpatients in Rehabilitation Department, and provide reference basis for high-risk fever patients to take positive and effective nursing measures.
\end{abstract}

\section{Keywords}

Rehabilitation Department, Fever, Risk Factors,

Least Absolute Shrinkage and Selection Operator,

Nursing Care
${ }^{\star}$ These authors contributed to the work equally and should be regarded as co-first authors.

${ }^{\#}$ Corresponding authors. 


\section{Introduction}

Body temperature is an objective reflection of the internal activities of the body and an important indicator of vital signs. The temperature of the human body is not immutable, when the human body is affected by certain factors, the body temperature will fluctuate accordingly. Fever is a non-specific response of the body to various stimulating factors [1], and there are many clinical factors leading to human fever, often involving dozens or even hundreds of diseases, therefore, it is not easy to make a clear identification of different cases in a short period of time. In recent years, the number of patients in the rehabilitation ward is increasing year by year, and most of the patients in the Rehabilitation Department come from other hospitals, with many complications, long hospitalization time, low immune function and low self-care ability [2]. All of these are easy to cause nosocomial infection and increase the risk of fever. Fever will lead to the disorder of the internal environment of the body, and even affect the prognosis of the disease, which brings great trouble to clinical treatment and nursing care.

Lasso retrospective analysis: in 1996, Robert Tibshirani proposed a new variable selection technique: Lasso, or Least Absolute Shrink age and Selection Operator [3]. The basic idea of Lasso regression is that under the condition that the sum of the absolute values of a regression coefficient is less than a constant, then the sum of squares of the residual is minimized and the unimportant regression coefficients are strictly compressed to zero, the corresponding variables are deleted, and finally the variable selection is realized [4]. Lasso algorithm features: variable screening and complexity adjustment while fitting the generalized linear model. This not only overcomes the limitation of stepwise regression variable selection method, but also retains the interpretability of subset selection and the stability of ridge regression, and the irrelevant factor coefficient can be reduced to 0 and eliminated. This algorithm is suitable for variable selection with multiple influencing factors [5] [6]. Because Lasso regression can effectively deal with the multiple collinearity problem in the model, this variable selection technique is used to explore the risk factors of fever in inpatients in Rehabilitation Department, which is helpful to identify patients with high risk of fever in early stage, so that nurses and doctors can take planned and purposeful prevention and treatment measures together in clinic, so as to promote patients' recovery as soon as possible.

\section{Method}

\subsection{Participant}

The study was conducted on the Rehabilitation Department of the First Affiliated Hospital of Jinan University from July 2019 to December 2019. The fever group included 51 patients and the non-fever group included 49 patients without fever. Exclusion criteria: 1) hospitalization days < 24 hours; 2) there were already patients with fever at the time of admission; 3) Fever caused by infectious diseases. 


\subsection{Data Collection}

The collection of medical records was completed by the same nurse who had a full-time bachelor's degree and worked more than 5 years in clinical nursing in the Rehabilitation Department. The investigation nurses comprehensively used the hospital electronic medical record information platform and the hospital laboratory examination system, and adopted the method of retrospective analysis to consult the in-patient medical records of the study subjects. Recorded the clinical information of the patient, including two parts: 1) the basic information of the patient: name, sex, age; 2) related risk factors that may lead to fever during hospitalization: length of stay, main diagnosis, With or without complications (cognitive impairment, dysphagia, diabetes, hypertension, respiratory tract infection, pressure sores/skin infection, kidney injury, surgical wound, etc.), past history (history of respiratory tract infection within half a year, history of abnormal urine test within half a year, history of tracheotomy, history of operation within one month), first course of disease, feeding patterns (oral feeding, assisted oral feeding, intermittent oral/nasal feeding, indwelling nasal feeding), Range of limb mobility (walking, need assistance, relying on wheelchair, lying in bed), urination (no incontinence, occasional incontinence, incontinence or need for catheterization), With or without central venous catheterization, laboratory indicators (urine routine, urine culture, sputum culture, blood culture, etc.), X-ray.

\subsection{Data Analysis}

Lasso regression analysis was used to analyze the clinical data, comorbidities, related laboratory values and possible risk factors of the two groups of patients.

\section{Results}

\subsection{Multi-Factor Prediction and Evaluation of Fever Risk Screening of Inpatients in Rehabilitation Department}

A total of 22 potential fever-related risk factors were included in this study, including basic demographic data, clinical information, laboratory values and so on. We applied least absolute shrinkage and selection operator (Lasso) regression to predict incident of fever, assessing ten-fold cross-validated area under the receiver operating characteristic curve (AUC) and 95\% CIs. In Figure 1, the representative related factors of fever are screened out. When the Lambda parameter is selected as the minimum, the cross-verification results show that: see Figure 1, the ordinate represents the target parameter, the lower coordinate represents $\log (\lambda)$, the upper abscissa is the number of non-zero coefficient variables in the model. For each $\lambda$ value, when the values of the target parameter were shown by the red dot, a confidence interval of the target parameter can be obtained. The two vertical lines in Figure 1 indicate two special $\lambda$ values: lambda.min and lambda.1se, respectively. Lambda.min means to obtain the $\lambda$ value of the mean value of the minimum objective parameter among all the $\lambda$ values; Lambda.1se means that lambda.min obtains the $\lambda$ value of the simplest model within a variance range. In this study, the value of lamb-da.1se was 0.7852 . The variables entering the model were pres- 
sure sore or skin infection, history of hypertension, current history of respiratory tract infection, feeding patterns, main diagnosis, history of respiratory tract infection within half a year, kidney damage, days of hospitalization and first course of disease. With the change of $\lambda$ value and the screening of model variables, Lasso regression is used to analyze the prediction probability and draw the working characteristic curve (Receiver Operator Characteristic curve, ROC). In Figure 2, each curve represents the changing trajectory of each independent variable coefficient. The ordinate is the value of the coefficient, the lower abscissa is log $(\lambda)$, and the upper abscissa is the number of non-zero coefficients in the model at this time. With the increase of $\lambda$ value, the greater the compression degree of the model, the less the number of independent variables entering the model, and the function of the model to select the main variables becomes stronger.

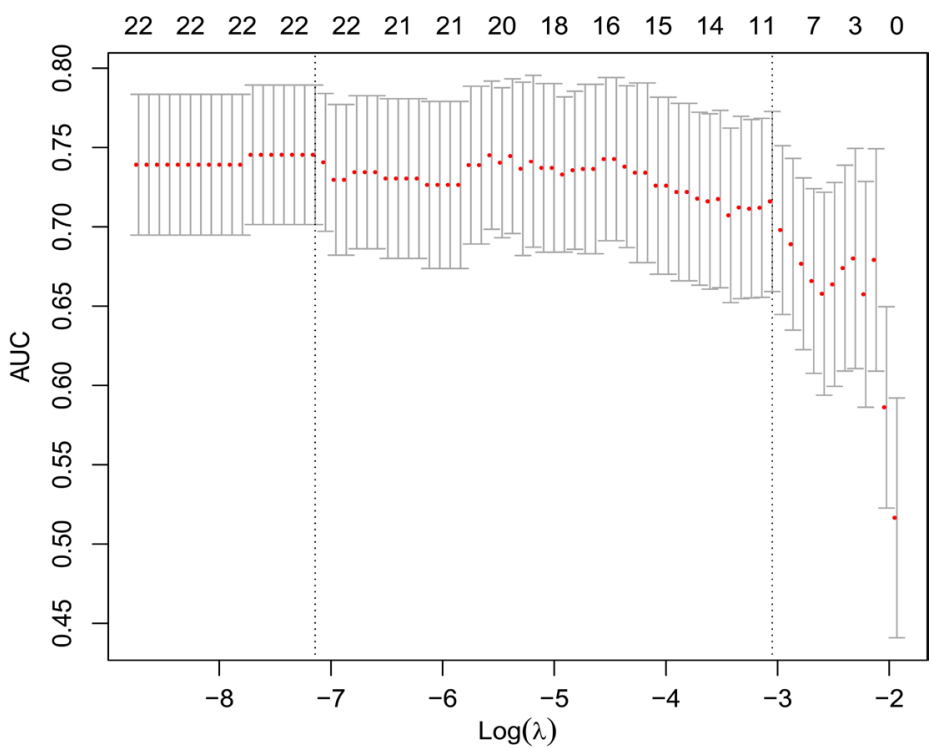

Figure 1. Texture feature selection.

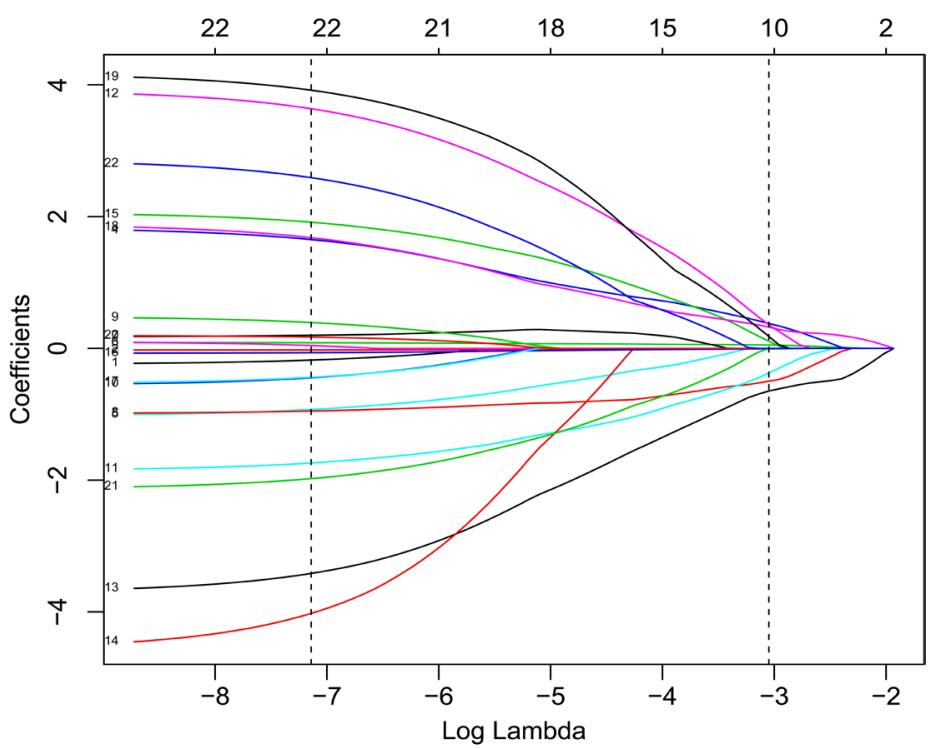

Figure 2. Lasso coefficient profiles. 


\subsection{Analysis of Risk Factors of Fever among Inpatients in Rehabilitation Department}

According to the results of Lasso variable selection, see Figure 3: pressure sore or skin infection $(\beta=0.647)$, history of hypertension $(\beta=0.497)$, current history of respiratory tract infection $(\beta=0.374)$, main diagnosis $(\beta=-0.378)$, history of aspiration tract infection within half a year $(\beta=-0.357)$, feeding patterns $(\beta=$ $-0.324)$, kidney damage $(\beta=-0.107)$, hospital stay $(\beta=-0.051)$, first course of disease $(\beta=0.006)$. Among them, pressure sore or skin infection, history of hypertension, current history of respiratory tract infection and feeding patterns were the higher risk factors of fever in inpatients in Rehabilitation Department. The first course of disease, main diagnosis, history of aspiration tract infection within half a year, kidney damage and hospitalization days were lower risk factors. The meaning of each variable assignment is shown in Table 1.

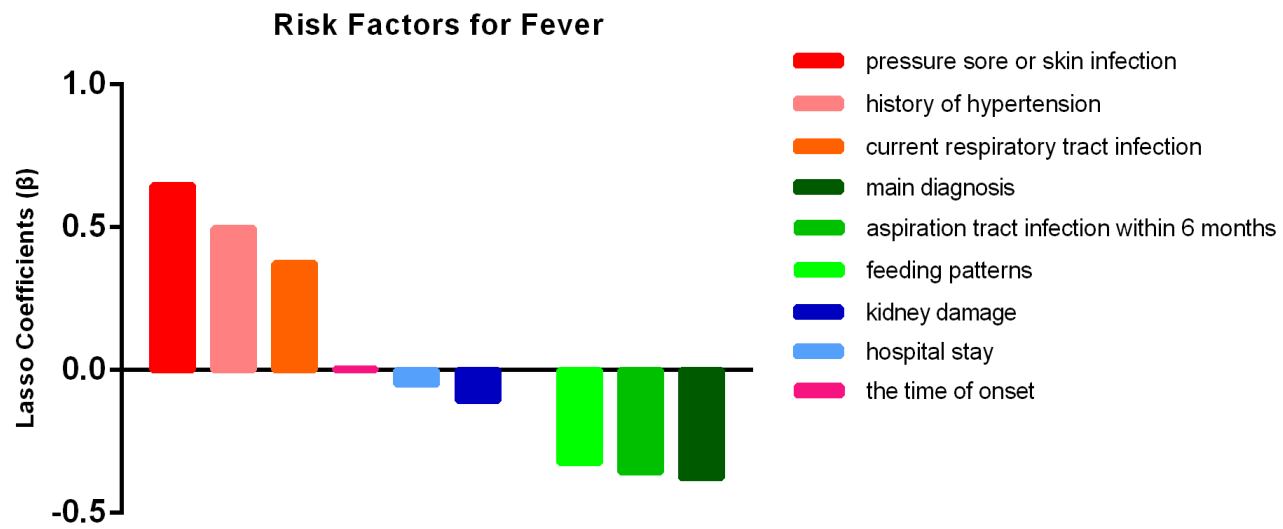

Figure 3. Coefficients of risk factors related to fever.

Table 1. Lasso regression analysis of related factors of fever among inpatients in Rehabilitation Department.

\begin{tabular}{ccc}
\hline \multicolumn{1}{c}{ Variables } & Assignment & Variable coefficient $\beta$ \\
\hline Pressure sores or skin infections & $1=$ yes, $2=$ no & 0.647 \\
History of hypertension & $1=$ yes, $2=$ no & 0.497 \\
Murrent history of respiratory tract infection & $1=$ yes, $2=$ no & -0.378 \\
Main diagnosis & $1=$ Brain damage, $2=$ Non-brain damage & -0.357 \\
History of respiratory tract infection within half a year & $1=$ yes, $2=$ no & -0.324 \\
Feeding patterns & $1=$ oral feeding, $2=$ assisted oral feeding, \\
Kidney damage & $1=$ yes, $2=$ no \\
Hospitalization days & $($ Continuous variable $)$ & -0.107 \\
The first course of disease & $1=$ yes, $2=$ no
\end{tabular}




\section{Discussion}

\subsection{To Explore the Importance of Risk Factors of Fever in Inpatients in Rehabilitation Department}

Fever is often a common nursing problem in clinical departments. Excessive body temperature or long-term fever will cause some physiological disorders of the patient. Such as gastrointestinal dysfunction, often with loss of appetite or with nausea, vomiting; abnormal nervous system function, high fever can cause irritability, delirium, hallucinations, convulsions and other changes in consciousness, in addition, fever will also consume a lot of material and energy, which can lead to weight loss, imbalance of water and electrolytes, and so on. These will lead to more complex conditions of the patients, and easily cause a variety of complications, prolong the rehabilitation time, increase the disability rate of the patients, and even threaten their life [7] [8]. Through the regression analysis of fever risk of inpatients in Rehabilitation Department by using Lasso, it is concluded that the higher the evaluation score is, the greater the risk of fever in hospital is. It can be seen that this study has a certain guiding significance for the identification of fever risk factors in Rehabilitation Department. Clinically, we should strengthen the early fever risk assessment of patients in the Rehabilitation Department in order to identify early patients with high risk of fever and take effective nursing measures to reduce the incidence of fever during hospitalization.

\subsection{Pressure Sore or Skin Infection Is a High Risk Factor for Fever among Inpatients in Rehabilitation Department}

The results of this study show that there is a correlation between pressure sores or skin infections and fever in inpatients in the Rehabilitation Department, that is, patients with pressure sores or skin infections are more likely to develop fever. Pressure sore is a common clinical complication, which increases the pain of patients and reduces their quality of life [9] [10]. Pressure sore or skin infection, with the expansion of the broken surface of the skin, cause secondary infection, blood culture, blood routine examination abnormalities, and even cause serious systemic infection and life-threatening. In this study, Lasso regression analysis showed that pressure sore or skin infection $(\beta=0.647)$ was a high risk factor. In short, pressure sore or skin infection increased the risk of fever in patients, and attention should be paid to the prevention and treatment of pressure sores in inpatients.

\subsection{Effects of Hypertension, Existing Respiratory Tract Infection and History of Respiratory Tract Infection within Half a Year on Fever of Inpatients in Rehabilitation Department}

In this study, hypertension and existing respiratory tract infection were high risk factors for fever among inpatients in Rehabilitation Department. Patients in the Rehabilitation Department often suffer from multiple diseases at the same time, often with diseases such as high blood pressure and/or respiratory infections. 
Hypertension is not only the most common chronic disease, but also the most important risk factor of cardio-cerebrovascular disease, which can lead to target organ damage. Stroke, myocardial infarction, heart failure and chronic kidney disease are the main complications, which lead to the complexity of the patient's condition and the decrease of autoimmunity [11]. Due to the decrease of resistance, patients are prone to nosocomial infection during hospitalization [12] [13]. Lasso regression analysis showed that there was a strong correlation between hypertension and the risk of fever $(\beta=0.497)$. Therefore, inpatients in Rehabilitation Department should pay attention to the evaluation and monitoring of hypertension.

The inpatients in the Rehabilitation Department are older, have more basic diseases, and are accompanied by varying degrees of dysfunction. Its low self-care ability, difficulty in expectoration, dysphagia and so on, increase the incidence of respiratory tract infection. In addition, inpatients in Rehabilitation Department have a high incidence of nosocomial infection, and respiratory tract infection is one of the main sites of infection among inpatients in Rehabilitation Department [14] [15]. Lasso regression analysis showed that inpatients in Rehabilitation Department had a relatively high risk of fever with existing respiratory tract infection $(\beta=0.374)$. It is suggested that more attention should be paid to the prevention and treatment of respiratory diseases. The history of respiratory infection within half a year is a low risk factor for fever in patients in the Rehabilitation Department, which may be due to the control of respiratory tract inflammation by recent clinical treatment, but the relationship between the history of respiratory infection and fever patients in the Rehabilitation Department within half a year is still difficult to determine, which needs to be confirmed by a large sample study.

\subsection{Feeding Patterns and Major Diagnosis May Also Increase the Risk of Fever among Inpatients in the Rehabilitation Department}

The primary diseases of patients admitted to the Rehabilitation Department are mainly brain injury and orthopedic surgery, and the primary disease is often serious, and the nervous system function will be accompanied by varying degrees of damage after the attack. Its complications, abnormal immune function, neurological deficit, invasive operation and so on will increase the risk of infection in patients [16] [17] [18] [19]. The dysphagia and cough reflex function of these patients is weakened, which is easy to cause aspiration and increase the risk of infection [20]. Tube feeding is an invasive operation, which can destroy the integrity of the mucous membrane of the body. Some studies have shown that the infection rate of patients with invasive operation is more than $50 \%$, which is much higher than that of patients without invasive operation [21] [22]. Lasso regression analysis showed that the feeding pattern was related to the risk of fever $(\beta=$ $-0.324)$, suggesting that the patients with severe dysphagia had a higher risk of fever. Therefore, attention should be paid to the evaluation of swallowing function of hospitalized patients, guiding patients to adopt appropriate feeding pat- 
terns and nursing care. With the continuous development of medical technology, the survival rate of stroke has been greatly improved. Most of the patients in the Rehabilitation Department are stroke convalescent patients, while some non-brain injury patients are mostly after orthopedic surgery, such as spinal cord injury and limb fracture. Orthopedic surgery is mostly emergency surgery, if it is an open fracture, it has the possibility of wound contamination and if the surgical wound is large, the postoperative bed rest time is long, and there are implants, it will increase the risk of nosocomial infection [23]. Lasso regression analysis showed that the main diagnosis was that non-brain injury patients in Rehabilitation Department had a higher risk of fever $(\beta=-0.378)$, which should be paid attention to.

\subsection{Limitations}

1) This study is a retrospective study of a single case in the Rehabilitation Department of Grade 3A Hospital for half a year. The sample size is small and there may be a deviation. In the future, the sample size needs to be expanded to verify the results. 2) There are many influencing factors and links of fever in hospital. In addition to the factors selected in this study, more possible influencing factors need to be included.

\section{Conclusion}

In this study, Lasso regression was used to analyze the risk factors of fever in inpatients in Rehabilitation Department. The results showed that pressure sore or skin infection, history of hypertension and current history of respiratory tract infection were positively correlated with fever in inpatients in Rehabilitation Department, and they were medium and low risk factors. In addition, Brain injury, history of aspiration tract infection within half a year, and diet were negatively correlated with fever in inpatients in Rehabilitation Department, which were low risk factors. In a word, the results of this trial can play an auxiliary role in predicting the risk of fever in inpatients in Rehabilitation Department.

\section{Acknowledgements}

This study was supported by grants from the National Natural Science Foundation of China (Grant NO. 81701847), Guangdong Medical Science and Technology Research Foundation (Grant NO. A2020362, A2019120), Natural Science Foundation of Hunan Province (Grant NO. 2017JJ3497).

\section{Conflicts of Interest}

The authors declare no conflicts of interest regarding the publication of this paper.

\section{References}

[1] Cheng, X.C., et al. (2013) Analysis of Disease of Hospitalized Older Patients with Acute Fever. Chinese Journal of Lung Diseases (Electronic Edition), 6, 154-156. (In Chinese) 
[2] Shou, X.Q. (2015) Analysis of the Composition and Characteristics of Inpatients in Rehabilitation Department. Journal of Traditional Chinese Medicine Management, 23, 144-145. (In Chinese)

[3] Tibshirani, R. (1996) Regression Shrinkage and Selection via the Lasso. Journal of the Royal Statistical Society. Series B: Methodological, 58, 267-288. https://doi.org/10.1111/j.2517-6161.1996.tb02080.x

[4] Efron, B., et al. (2004) Rejoinder to "Least Angle Regression" by Efron et al. Annals of Statistics, 32, 494-499. https://doi.org/10.1214/009053604000000067

[5] Cheng, J., et al. (2018) Predictors of Breast Cancer Screening Utilization among Female at High Risk of Developing Breast Cancer: Application of a Lasso Logistic Model. Chinese Journal of Disease Control \& Prevention, 22, 551-554, 559. (In Chinese)

[6] Zhong, J.H. (2013) An Empirical Study on the Influencing Factors of Shanghai's Economic Growth Based on Lasso Method. Statistics \& Decision, No. 1, 154-156. (In Chinese)

[7] Shen, H.P., et al. (2016) Clinical Characteristics and Risk Factors of Nosocomial Infections in Elderly Patients with Stroke. Zhejiang Medical Journal, 38, 836-838. (In Chinese)

[8] Ma, L.Z., et al. (2016) Incidence of Pulmonary Infection and Correlation with Deep Venous Thrombosis in Elderly Stroke Patients. Chinese Journal of General Practice, 14, 2034-2036. (In Chinese)

[9] Ding, Y.M. and Wang, L. (2013) Guidance on Nursing Care of Pressure Sores in China. Professional Committee of Stoma, Wound and Incontinence Nursing of Chinese Nursing Association, Beijing, 45. (In Chinese)

[10] Fan, X.Y. and Huan, X.Q. (2011) Prevention and Nursing of Pressure Sore Compli-cated with Infection. Chinese Journal of Misdiagnostics, 11, 7768. (In Chinese)

[11] Liu, L.S. (2010) Chinese Guidelines for the Management of Hypertension. Chinese Journal of the Frontiers of Medical Science (Electronic Version), 19, 701-743. (In Chinese)

[12] Menendez, R., et al. (2003) Duration of Length of Stay in Pneumonia: Influence of Clinical Factors and Hospital Type. European Respiratory Journal, 22, 643-648. https://doi.org/10.1183/09031936.03.00026103

[13] File, T.J. and Tan, J.S. (2005) Pneumonia in Older Adults: Reversing the Trend. JAMA, 294, 2760-2763. https://doi.org/10.1001/jama.294.21.2760

[14] Zhao, K.Z. (2015) Analysing the Infection Factors and Countermeasures of Hospital Infection in Inpatients of the Department of Rehabilitation. E E-Journal of Translational Medicine, 2, 152-153+155. (In Chinese)

[15] Zhou, Y., Zhang, Y. and Zhou, L. (2019) Analysis of Bacteria and Drug Resistance of Nosocomial Infection in Rehabilitation Department. Journal of Traditional Chinese Medicine Management, 27, 37-38. (In Chinese)

[16] Lynch, E.A., et al. (2015) Rehabilitation Assessments for Patients with Stroke in Australian Hospitals Do Not Always Reflect the Patients' Rehabilitation Requirements. Archives of Physical Medicine and Rehabilitation, 96, 782-789. https://doi.org/10.1016/j.apmr.2014.12.009

[17] Shen, M.H., et al. (2019) Incidence and Risk Factors for Healthcare-Associated Infection in Hospitalized Patients in Rehabilitation Center. Chinese Journal of Infection Control, 18, 964-968. (In Chinese)

[18] LI, Y.T., et al. (2019) Correlation between Muscle Strength and Nosocomial Infec- 
tion in Stroke Patients Undergoing Surgery. Chinese Journal of Nosocomiology, 29, 1371-1373. (In Chinese)

[19] Hong, T., et al. (2017) Etiology and Risk Factors of Nosocomial Infections in Elderly Patients with Stroke. Chinese Journal of Nosocomiology, 27, 4409-4411+4426.

[20] Wang, Y.N. (2016) Preventive Effect of Early Dysphagia Management Measures on Pneumonia in Patients with Cerebral Apoplexy. E-Journal of Translational Medicine, 3, 71-72. (In Chinese)

[21] Huang, X.Z., et al. (2016) Clinical Characteristics of Hospital Infections in Patients with Acute Stroke. Chinese Journal of Nosocomiology, 26, 3211-3212 + 3227. (In Chinese)

[22] Liang, J.H., et al. (2015) Epidemiological Survey of Nosocomial Infections and Analysis of Risk Factors. Chinese Journal of Nosocomiology, 25, 2015-2017. (In Chinese)

[23] Wu, L.H., et al. (2018) Related Factors for Postoperative Nosocomial Infection in Patients of Orthopedics Department. Chinese Journal of Nosocomiology, 28, 3120-3123. (In Chinese) 